\title{
Bilateral horizontal gaze palsy in an 8-year-old girl; a rare case with NDUFS4 gene mutation: a case report and literature review
}

Mohammad Vafaee-Shahi ( $\sim$ dr.vafaeeshahi@gmail.com )

Iran University of Medical Sciences https://orcid.org/0000-0002-5901-3485

Saeide Ghasemi

Iran University of Medical Sciences

Mehran Beiraghi Toosi

Mashhad University of Medical Sciences

Mahmoud Reza Ashrafi

Tehran University of Medical Sciences

Reza Shervin Badv

Tehran University of Medical Sciences

Alireza Tavasoli

Tehran University of Medical Sciences

Leila Tahernia

Tehran University of Medical Sciences

Case report

Keywords: Leigh syndrome, gaze palsy, lactate, NDFUS4 gene, mitochondria

Posted Date: March 22nd, 2019

DOI: https://doi.org/10.21203/rs.2.481/v1

License: (c) (i) This work is licensed under a Creative Commons Attribution 4.0 International License.

Read Full License 


\section{Abstract}

Background: Leigh syndrome (LS) is a rare and inherited disease which is associated with progressive neurological disorders. The molecular underlying mechanism in LS is defined with some defects in mitochondrial respiratory chain enzymes. Case presentation: Here, an 8-year-old girl is reported with bilateral horizontal gaze palsy, ataxia and drowsiness. She developed unsteady gait, drowsiness, progressive ataxia and intention tremor during her admission period. The laboratory tests were reported within normal values including biochemical, hematological, immunological, infectious and inflammatory markers and blood and cerebrospinal fluid (CSF) lactate. Brain magnetic resonance imaging (MRI) demonstrated dorsal midbrain, bilateral putamen nuclei and cerebellar dentate nucleus involvement. Ocular examination revealed retinal atrophy and pale disk in both sides. These symptoms were in favor of a neurodegenerative disorder. Magnetic resonance spectroscopy (MRS) revealed an elevated lactate peak in involved areas which suggested a mitochondrial disease. Finally, the molecular genetic test reported NDUFS4 gene mutation which confirmed the presence of Leigh syndrome. She responded significantly to mitochondrial treatment cocktail and clinical signs and symptoms improved gradually. NDFUS4 gene encodes a subunit of mitochondrial complex I (NADH: ubiquinone oxidoreductase) that removes electrons from NADH and transfers them to the electron acceptor ubiquinone. Conclusion: Our findings indicated that various symptoms and clinical features can be found in Leigh syndrome which could be probably due to different mutations in mitochondrial genes. Therefore, appropriate clinical and laboratory settings along with brain MRI, MRS and genetic test analysis would be necessary for the early diagnosis.

\section{Background}

Leigh syndrome (LS) is a rare and inherited heterogeneous disease which is associated with progressive neurodegenerative brain damage. Diarrhea, vomiting and dysphagia are usually the initial signs of Leigh syndrome in infants. Clinically, it may be also associated with multiple features, including brainstem symptoms, ataxia, hypotonia, dystonia, respiratory insufficiency, pyramidal signs, lactic acidosis in blood or cerebrospinal fluid and hypertrophic cardiomyopathy (1). It is usually associated with cerebral hypotonia, movement and mental disabilities and respiratory failure which results in death within 2-3 years (2). Weakness of limbs and loss of sensation are also common in these cases (3). Although the basic neuropathological features in affected children are relatively similar, there is heterogeneity in clinical, genetic and biochemistry findings. Therefore, early diagnosis of the disease is essential to increase survival in affected patients.

The molecular mechanism underlying LS pathogenesis is mainly due to the defects in mitochondrial respiratory chain enzymes (4). Recent evidences have revealed that mutations in genes for the pyruvate dehydrogenase complex, ATP synthase subunit-6, cytochrome-c oxidase, and subunits of mitochondrial complex-I are responsible for LS $(4,5)$. NADH: ubiquinone oxidoreductase, encoded by NDUFS4 gene, is a subunit of mitochondrial complex-I which catalyzes the oxidation of NADH (6). Here, this is the first case of LS from Iran with NDFUS4 gene mutation presented with horizontal gaze palsy. 


\section{Case Presentation}

An 8-year-old girl was admitted to Rasool Akram hospital with bilateral horizontal gaze palsy, ataxia and drowsiness. The gaze palsy was initiated in the right eye during the last year and then gradually affected the left eye during the previous month. She developed unsteady gait, drowsiness, progressive ataxia, intention tremor and seizure during the admission time. The past medical history of the patient revealed that she was the first child of the family, from a consanguineous marriage. She had also developmental milestone delay. She had the history of seizure around 4 years ago, without current medication therapy.

The vital signs were stable during her admission time. She was confused; however, she responded to verbal stimulations. Horizontal gaze palsy was detected in both eyes under ocular examinations, without any vertical gaze palsy. Nystagmus was not observed during the examination .The normal light reflection was detected in her pupils. Ocular examination revealed retinal atrophy and pale disk in both sides. Cranial nerve examination was normal. Deep tendon reflexes (DTR) were Brisk. Cerebellar examination revealed positive tandem gait, intention tremor, ataxic and wide based gait. The results of finger-to-nose and heel to shin tests were detected normal. The biochemical test results, complete blood count (CBC) and electrolytes were reported within the normal ranges. Dorsal midbrain involvement were reported in brain Magnetic resonance imaging (MRI) (Figure 1), which was provided one month ago out of our center. Total abdominal and pelvic ultrasounds were performed in order to rule out the possibility of organomegaly and malignancy. Electroencephalography (EEG) was performed showing slowing background activity prominent in posterior area without epileptiform discharges.

On the 5th day of admission, the patient presented a generalized tonic-clonic (GTC) seizure lasted for one minute. Cerebrospinal fluid (CSF) was analyzed for cells, biochemical tests, lactated dehydrogenase $(\mathrm{LDH})$, and lactate contents that the results were reported acceptable. Anti- Neuromyelitis optica antibody (NMO), IgG index and oligoclonal bands (OCB) tests were performed for the possibility of autoimmune diseases. A basic metabolic panel test was performed for the assessment of serum ammoniac, lactate, pyruvate, and amino acids content with high performance liquid chromatography (HPLC) method. The possibility of specific metabolic syndromes was ruled out with all normal metabolic results. The patient received biotin, Vitamin B1, B12 and Vitamin E to evaluate the possibility of Wernicke-Korsakoff syndrome. However, no change in signs and symptoms was observed following these treatments. Serum immunoglobulins were evaluated in order to rule out the possibility of ataxia telangiectasia and other immune deficiencies. The laboratory test results including ANA; Anti-ds-DNA; serum complements (C3, C4, C5), SSA-Ro, SSA-LA; RPR; AFP; Anti-GM Ab (IgM and IgA); 32 glycoproteins; Anti-phospholipids; Anticardiolipin (IgM and IgG); HLA (B5 and B51); and serum ACE were within normal values. Therefore, the possibility of neuro-Behcet disease, lupus erythematous, neuro-brucellosis, and neuro-sarcoidosis was declined by detecting laboratory results. The infectious diseases biomarkers were performed to rule out the possibility of infectious diseases including HIV Ab, VDRL, Anti-toxoplasma Ab (IgM and IgG), wright and 2ME. Wilson's disease was evaluated by 24-hour urine copper test. Serum levels of vitamin B12 and folic acid were normal. Listeria infection was a recommended diagnosis due to midbrain involvement. So, Ampicillin and Gentamycin was then started empirically. 
On the 10th day of admission, the second brain MRI was performed with and without contrast for the second time (Figure 2). Abnormal high signal flair and T2 lesions were detected in medulla, Midbrain, bilateral putamen nuclei and cerebellar dentate nucleus. Corticosteroid pulse therapy was started to resolve involved areas, increased signal in midbrain, progressive ophthalmoplegia and consequently the possibility of inflammatory lesions.

On the 15th day of admission, Magnetic Resonance Spectroscopy (MRS) demonstrated an elevated lactate peak in involved areas which could indicate the possibility of mitochondrial disease (Figure 3). Mitochondrial treatment cocktail (including Vitamin B2, Vitamin B6, folic acid, L-carnitine and coenzyme Q) was prescribed for the patient. Gaze palsy of the eyes, ataxia, tremor and ophthalmoplegia were improved relatively. The patient was discharged with mitochondrial treatment cocktail and oral prednisolone $(1 \mathrm{mg} / \mathrm{kg} /$ day). The patient was examined again two weeks after discharge. The complete improvement was observed in eye movements and gaze palsy; however, a mild intention tremor and ataxia were detected during the examination time. Whole exome sequencing was done to evaluate any genetic disorder. Finally, the molecular genetic test result showed mutation in NDUFS4 gene, confirming the diagnosis of Leigh syndrome.

\section{Discussion And Conclusion}

Leigh syndrome is a rare progressive neurological disorder typically in childhood that can be inherited as $X$-linked recessive, autosomal recessive and mitochondrial trait. The incidence of this syndrome is estimated approximately 2 in 100,000 births. Leigh syndrome may present early in life with multiple characteristics such as weakness, drowsiness, dystonia, psychomotor regression, cerebral hypotonia, ataxia, developmental milestones delay, and signs of brainstem involvement, seizures, visual loss and tachypnea. Some studies reported the occurrence of sleep apnea syndrome and abdominal symptoms in these patients (7). However, the exact mechanism is unknown. Progressive respiratory failure is one of the main leading causes of mortality among LS cases (8). Elevated blood and CSF lactate, as well as pyruvate levels can be found upon laboratory tests analysis. However, neuroimaging is a valuable tool for the diagnosis of Leigh syndrome (9).

The present case was referred to Rasool Akram Hospital with the chief complaint of progressive gaze palsy and also ataxia, intention tremor and drowsiness. Her ocular examination revealed retinal atrophy and pale disk. In a recent study, Jabeen et al., (10) reported a 37-year-old female with Leigh syndrome who admitted with similar ocular symptoms including bilateral horizontal gaze palsy and ataxic gait. The ocular involvement is frequently reported in patients with Leigh syndrome. Many patients with Leigh syndrome may suffer from ophthalmoplegia, a condition which is associated with weakness or paralysis of the muscles responsible for eyes movement (11). Some studies have reported nystagmus, optic atrophy, ptosis, strabismus, and pigmentary retinopathy in patients with Leigh syndrome $(11,12)$. Our case had also a past medical history of delayed development and seizure. Abnormal cerebellar test results were detected including abnormal tandem gait, intention tremor and ataxic gait. Most of the reported LS patients like the present case excluded infections, autoimmune diseases and toxins as the 
causes of presented symptoms (12). The second brain MRI reported dorsal midbrain, cerebellar dentate nucleus and bilateral putamen nuclei involvement. These symptoms pointed towards a neurodegenerative disorder. A basic metabolic panel test result showed normal serum ammonia, pyruvate, and amino acids contents and normal blood and CSF lactate. Magnetic resonance spectroscopy revealed an elevated lactate peak in involved areas which suggested a possible mitochondrial disease. Eventually, the molecular genetic test result revealed NDUFS4 gene mutation which confirmed the diagnosis of Leigh syndrome. She responded significantly to mitochondrial treatment cocktail and significant improvement was observed in clinical symptoms. Several studies showed similar responses to mitochondrial treatment cocktail in different cases (10). Goldenberg et al., (7) reported a 22-year-old woman with Leigh syndrome who had partial deficiency in cytochrome $\mathrm{C}$ oxidase. They observed excellent response to mitochondrial treatment cocktail.

Molecular genetic studies illustrated that Leigh syndrome can be resulted from defects or mutations in mitochondrial enzymes such as pyruvate dehydrogenase (PDHA1 gene mutations) (13), pyruvate carboxylase (SURF1 gene mutations in nuclear genome), ATP synthase subunit-6, cytochrome $C$ oxidase, and subunits of complex-I (14). Recent evidences identified 24 known mutations in mitochondrial genes and 21 in nuclear genes in Leigh syndrome (15). Here, we found NDUFS4 gene mutation which was associated with Leigh syndrome. This gene is a nuclear-encoded accessory subunit of mitochondrial complex I (NADH: ubiquinone oxidoreductase). This complex removes electrons from NADH and transfers them to the electron acceptor ubiquinone. Therefore, mutations in NDUFS4 gene can cause mitochondrial respiratory chain deficiencies. In another study, Leshinsky-Silver et al. (16) reported a case with Leigh syndrome due to NDUFS4 mutation. The patient developed predominant brainstem involvement. More recently, Ortigoza-Escobar et al., (17) reported a patient with NDUFS4 gene mutation related to Leigh syndrome.

These data has suggested that various symptoms and clinical features could be found in Leigh syndrome. Variations in the disease severity and the number of clinical features are probably due to different mutations in mitochondrial genes. Therefore, further studies are necessary to evaluate the relationship between genotype and phenotype. Our patient was born from parents with consanguineous marriage; however, no evidence of similar conditions was reported in his family. Since consanguineous marriage is common in Iran, there may be a relationship between the incidence of Leigh syndrome and consanguineous marriage. Therefore, further considerations are required to evaluate the correlation. Therefore, premarital genetic counseling and education may be helpful.

Leigh syndrome is a rare mitochondrial disease which is associated with progressive neurological disorders. Our case presented with progressive bilateral gaze palsy, ataxia, retinal atrophy, pale disk, unsteady gait, intention tremor, and drowsiness. The brain MRI showed dorsal midbrain and cerebellar dentate nucleus involvement. These symptoms pointed towards a neurodegenerative disorder. MRS illustrated an elevated lactate peak in involved area despite normal blood and CSF lactate which indicated a mitochondrial disease. The genetic test analysis revealed NDUFS4 gene mutation which confirmed the diagnosis of Leigh syndrome. A good response was detected to mitochondrial treatment 
cocktail and the clinical signs and symptoms improved by treatment. Our findings indicated that various symptoms and clinical features can be found in Leigh syndrome which could be probably due to different mutations in mitochondrial genes. Therefore, appropriate clinical and laboratory settings along with brain MRI, MRS and genetic test analysis would be necessary for the early diagnosis. Our patient was born from parents with consanguineous marriage, which highlighted the possible relationship between familial marriage and incidence of Leigh syndrome. Since consanguineous marriage is popular in Iran, premarital genetic counseling and education may be helpful.

\section{Abbreviations:}

Leigh syndrome (LS), Cerebrospinal fluid (CSF), Magnetic resonance imaging (MRI), Magnetic resonance spectroscopy (MRS), Nicotinamide adenine dinucleotide hydrogen (NADH), Deep tendon reflex (DTR), Complete blood count (CBC), Electroencephalogram (EEG), Generalized tonic clonic (GTC), Lactate dehydrogenase (LDH), Anti-Neuromyelitis optica antibody (NMO-Ab), High performance liquid chromatography (HPLC), Anti nucleotide antibody (ANA), Anti- double stranded DNA (Anti-ds DNA), Antisjögren's- syndrome- related antigen A (Anti-SSA-RO and SSA-LA), Rapid Plasma Reagin (RPR), Alpha fetoprotein (AFP), Anti-gangelioside antibody (anti-GM antibody), Serum Angiotensin converting enzyme (ACE), Human immunodeficiency virus (HIV), Venereal disease research laboratory test (VDRL), 2mercaptoethanol Brucella agglutination test (2ME).

\section{Declarations}

\section{Ethics approval and consent to participate:}

This case study was accredited by Ethical Committee of Iran University of Medical Sciences. Written informed consent was obtained from the parents of the patient for publication of this Case Report and any accompanying images. A copy of the written consent is available for review by the Editor of this journal.

\section{Consent for publication:}

The consent for publication of personal detailed data was obtained from parents.

\section{Availability of data and material:}

The datasets used during the current case study are available from the corresponding author on reasonable request.

\section{Competing interests:}

The authors declare no conflict of interest in preparing this case presentation.

\section{Funding:}


This case presentation received no specific grant from any funding agency in the public, institutions or not-for-profit sectors.

\section{Authors' contributions:}

$\mathrm{VM}, \mathrm{AM}, \mathrm{BM}, \mathrm{BR}$ and $\mathrm{TA}$ analyzed and interpreted the patient data regarding the neurologic disease. TL and GS performed follow up the patient and were major contributors in writing the manuscript. All authors read and approved the final manuscript.

\section{Acknowledgements:}

Not applicable

\section{References}

1. Shrikhande DY, Kalakoti P, Aarif Syed MM, Ahya K, Singh G. A rare mitochondrial disorder: Leigh sydrome - a case report. Ital J Pediatr. 2010;36:62.

2. Topçu M, Saatci I, Anil Apak R, Söylemezoglu F, Akçören Z. Leigh Syndrome in a 3-Year-Old Boy with Unusual Brain MR Imaging and Pathologic Findings. American Journal of Neuroradiology 2000;21:224-7.

3. Lake NJ, Bird MJ, Isohanni P, Paetau A. Leigh syndrome: neuropathology and pathogenesis. J Neuropathol Exp Neurol. 2015;74:482-92.

4. Sofou K, et al. A multicenter study on Leigh syndrome: disease course and predictors of survival. Orphanet Journal of Rare Diseases. 2014;9:52.

5. Shrikhande DY, Kalakoti P, Aarif Syed MM, Ahya K, Singh G. A rare mitochondrial disorder: Leigh syndrome - a case report. talian Journal of Pediatrics. 2010;62:36.

6. Scacco S, et al. Pathological Mutations of the Human NDUFS4 Gene of the 18-kDa (AQDQ) Subunit of Complex I Affect the Expression of the Protein and the Assembly and Function of the Complex. The Journal of Biological Chemistry. 2003;278:44161-7.

7. Goldenberg PC, Steiner RD, Merkens LS, Dunaway T, Egan RA, Zimmerman EA, et al. Remarkable improvement in adult Leigh syndrome with partial cytochrome c oxidase deficiency. Neurology. 2003;60:865-8.

8. Barkovich AJ. Toxic and metabolic brain disorders. In:Pediatric Neuroimaging. New York: Raven Press. 1995:55-106.

9. Hombal AG, Narvekar VN. Leigh's Disease (Subacute Necrotising Encephalomyelopathy) - A Case Report. Ind J Radiol Imag. 2005;15:217-9. 
10. Jabeen SA, Sandeep G, Rukmini Mridula K, Meena AK, Borgohain R, Sundaram C. Adult-onset Leigh's disease: A rare entity. Ann Indian Acad Neurol. 2016;19:140-2.

11. Grönlund MA, Andersson S, Åkebrand R, Seyedi Honarvar AK, Sofou K, Darin N, et al. Ophthalmologic involvement in Leigh syndrome. Acta Ophthalmologica 2017;95:e76-e.

12. Han J, Lee YM, Kim SM, Han SY, Lee JB, Han SH. Ophthalmological manifestations in patients with Leigh syndrome. Br J Ophthalmol. 2015;99:528-35.

13. Matthews PM, Marchington DR, Squier M, Land J, Brown RM, Brown GK. Molecular genetic characterization of an X-linked form of leigh's syndrome. Ann Neurol. 1993;33:652-5.

14. Moslemi AR, Darin N. Molecular genetic and clinical aspects of mitochondrial disorders in childhood. Mitochondrion. 2007;7:241-52.

15. Finsterer J. Leigh and Leigh-like syndrome in children and adults. Pediatr Neurol. 2008;39:223-35.

16. Leshinsky-Silver E, Lebre AS, Minai L, Saada A, Steffann J, Cohen S, et al. NDUFS4 mutations cause Leigh syndrome with predominant brainstem involvement. Mol Genet Metab. 2009;97:185-9.

17. Ortigoza-Escobar JD, et al. Ndufs4 related Leigh syndrome: A case report and review of the literature. Mitochondrion. 2016;28:73-8.

\section{Figures}



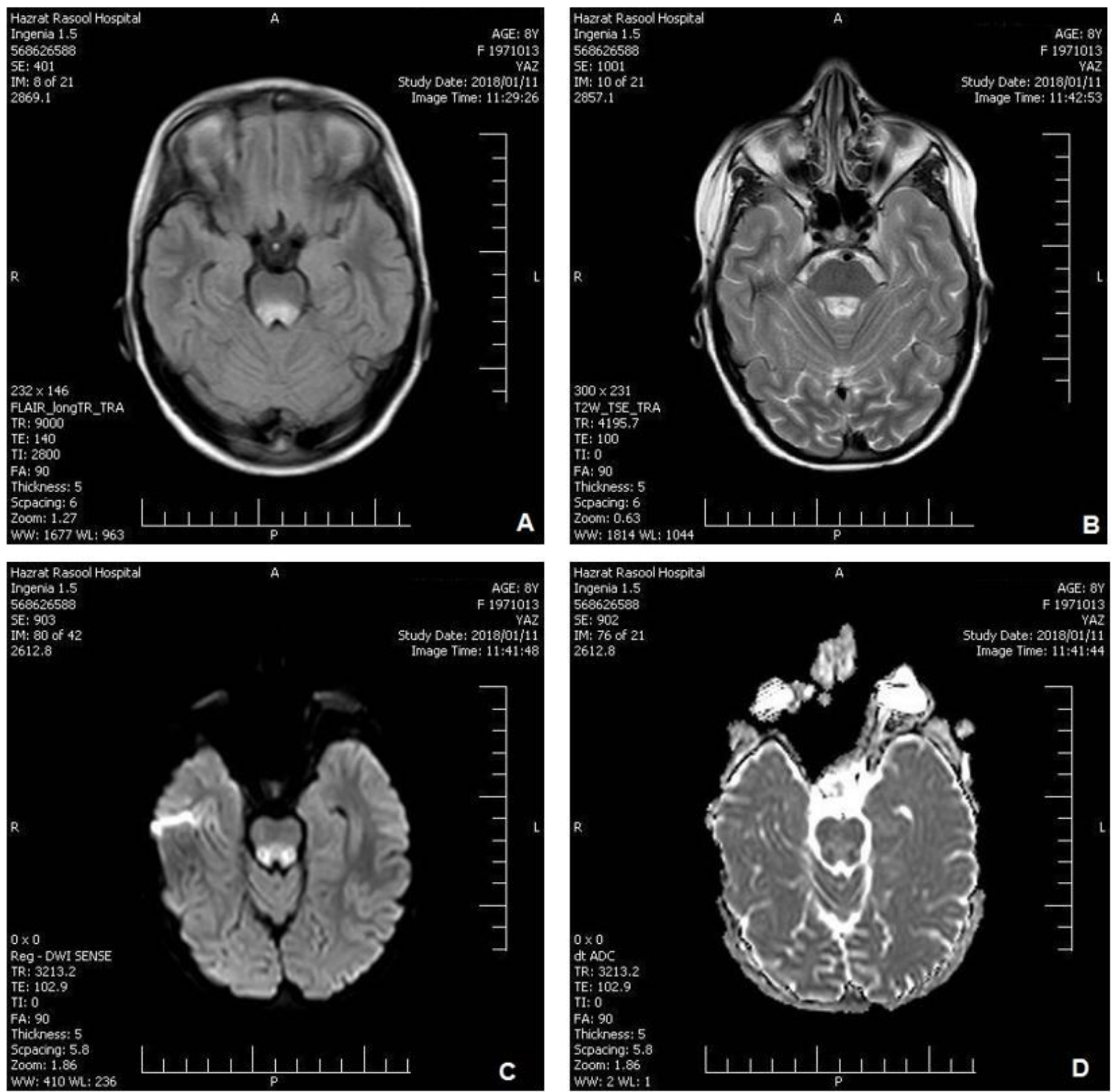

Figure 1

The first brain MRI. (A): Axial brain MRI (flair) showing hyper intensity in dorsal midbrain; (B): Axial brain MRI (T2) showing hyper intensity in dorsal midbrain; (C): Axial brain MRI (DWI) showing hyper intensity in dorsal midbrain; (D): Axial brain MRI (ADC map) showing restriction in dorsal midbrain involvement 

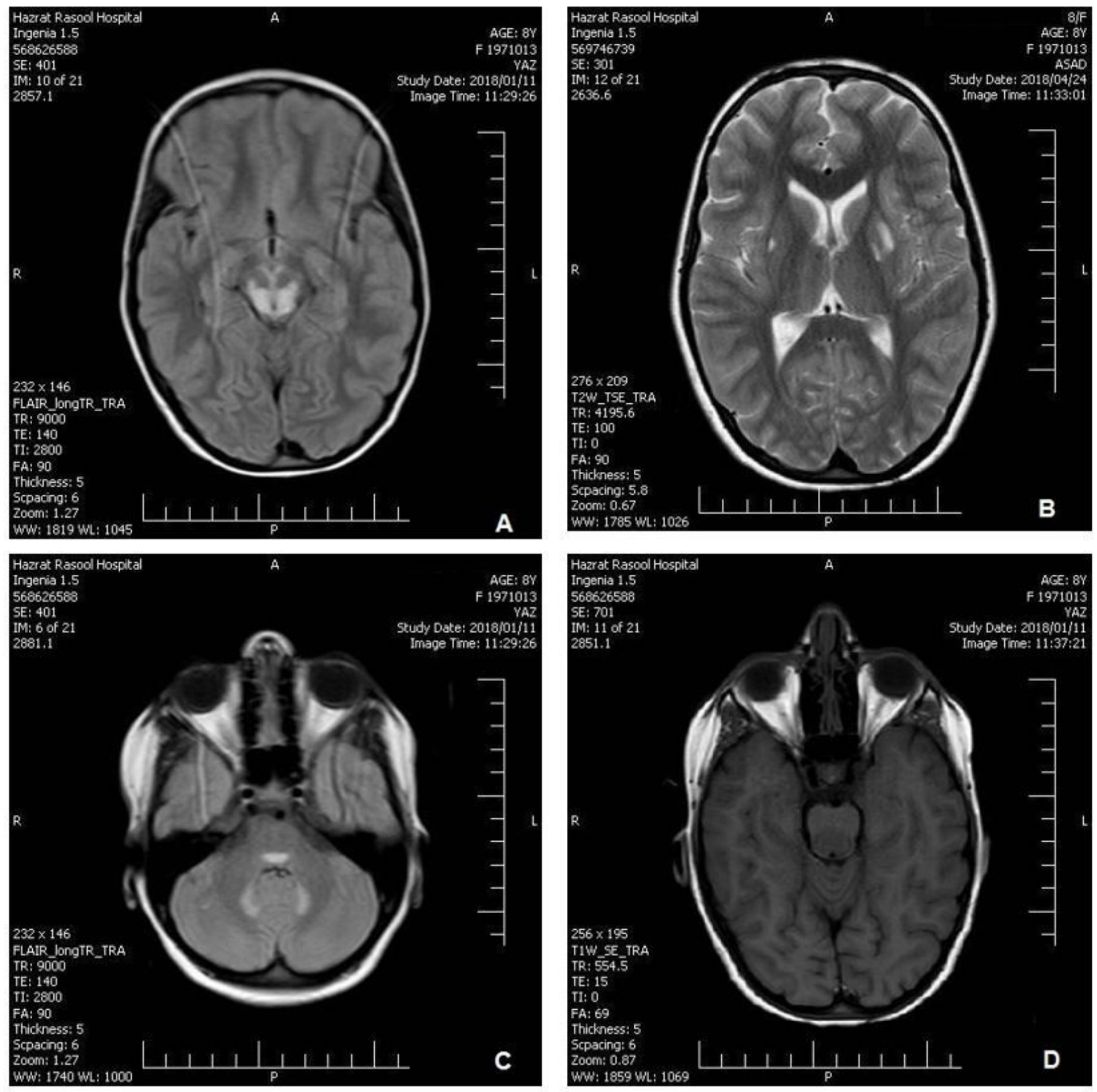

Figure 2

The second brain MRI. (A): Axial brain MRI (flair) showing hyper intensity in dorsal midbrain; (B): Axial brain MRI (T2) showing bilateral hyper intensity in putamen nucleus; (C): Axial brain MRI (flair) showing hyper intensity in dorsal midbrain and dentate nucleus of cerebellum; (D): Axial brain MRI (T1) showing hypo intensity in dorsal midbrain 


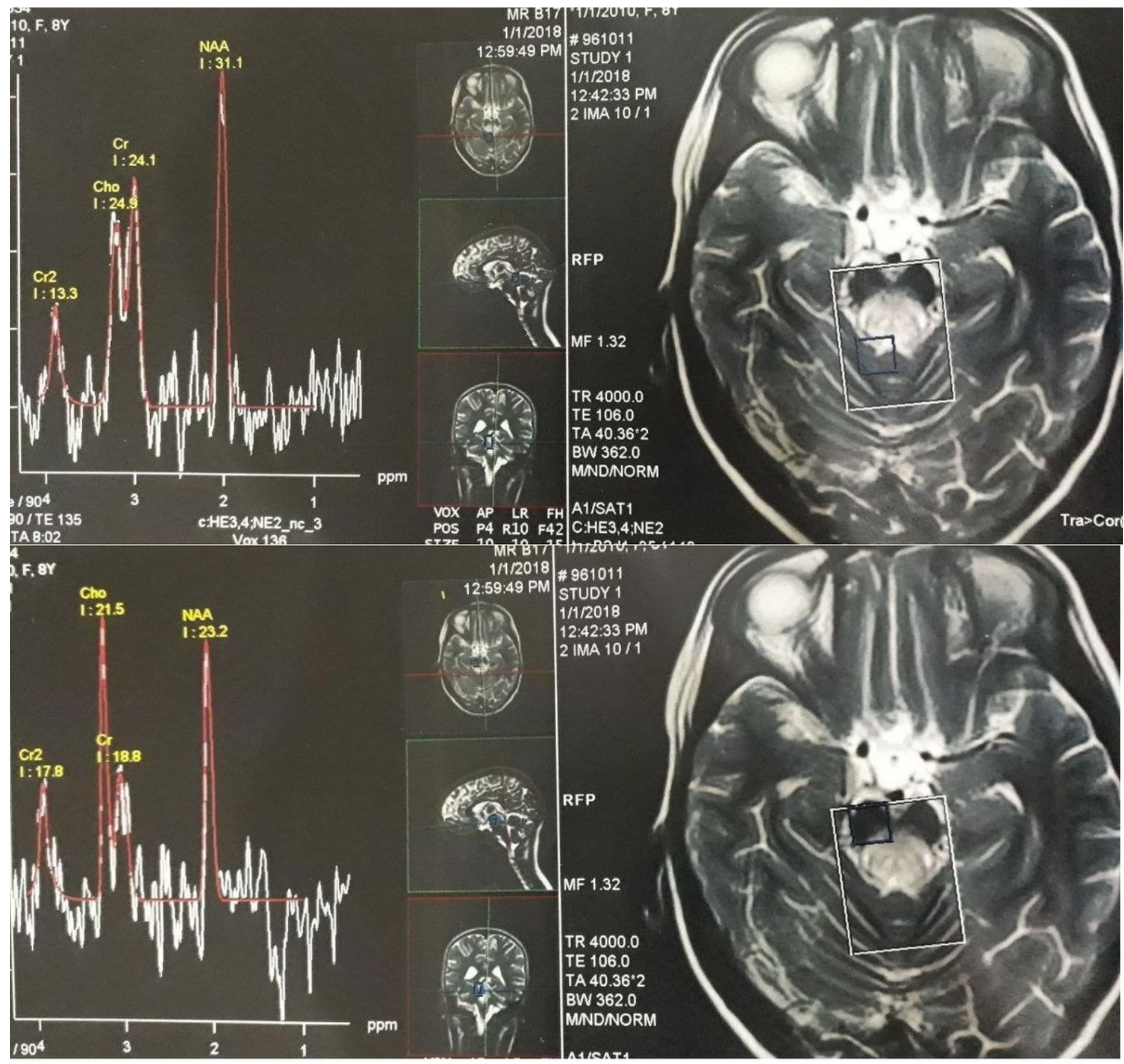

Figure 3

Magnetic resonance spectroscopy result. The result demonstrated an elevated lactate peak in involved areas

Supplementary Files

This is a list of supplementary files associated with this preprint. Click to download.

- supplement1.docx 